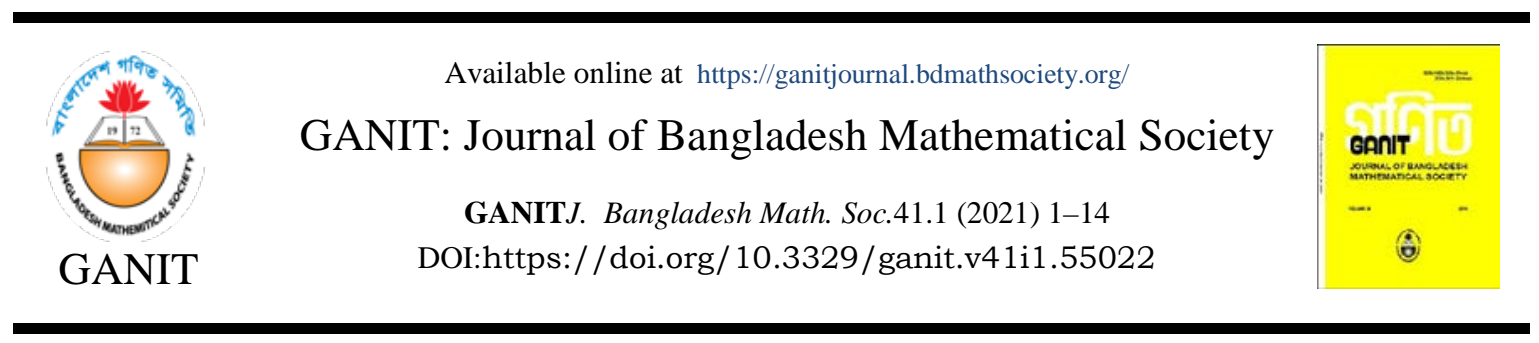

\title{
Galerkin Approximations for the Solution of Fredholm Volterra Integral Equation of Second Kind
}

\author{
Asma Akter Akhi ${ }^{\mathrm{a}}$, and Goutam Saha ${ }^{\mathrm{a}}$, \\ ${ }^{a}$ Department of Mathematics, University of Dhaka, Dhaka-1000, Bangladesh.
}

\begin{abstract}
In this research, we have introduced Galerkin method for finding approximate solutions of Fredholm Volterra Integral Equation (FVIE) of 2nd kind, and this method shows the result in respect of the linear combinations of basis polynomials. Here, BF (product of Bernstein and Fibonacci polynomials), $\mathrm{CH}$ (product of Chebyshev and Hermite polynomials), CL (product of Chebyshev and Laguerre polynomials), FL (product of Fibonacci and Laguerre polynomials) and LLE (product of Legendre and Laguerre polynomials) polynomials are established and considered as basis function in Galerkin method. Also, we have tried to observe the behavior of all these approximate solutions finding from Galerkin method for different problems and then a comparison is shown using some standard error estimations. In addition, we observe the error graphs of numerical solutions in Galerkin method for different problems of FVIE of second kind.
\end{abstract}

(C) 2021 Published by Bangladesh Mathematical Society

Received: September 05, 2020 Accepted: January 04, 2021 Published Online: August 04, 2021

Keywords: Fredholm Volterra Integral Equation; BF, CH, CL, FL and LLE polynomials; Galerkin Method. AMS Subject Classification 2020: 45A05; 65R20; 68W05

\section{Introduction}

Integral equation is a key mathematical perceptive in the area of mathematics, and it has vast applications in physics, biology, economics, signal processing, engineering and other fields of science. Many ordinary and partial differential equations associated with different initial and boundary values can be reduced to different integral equations. There are various types of integral equations, among them FIEs and VIEs are the main two classes of integral equations. Both of these have first and second kind, linear and nonlinear form.

\begin{tabular}{|l|}
\hline Nomenclature \\
\hline BF $\quad$ product of Bernstein and Fibonacci polynomials \\
\hline
\end{tabular}

\footnotetext{
* Corresponding author: Goutam Saha,E-mail address: gsahamath@du.ac.bd
} 

$\mathrm{CH} \quad$ product of Chebyshev and Hermite polynomials
CL product of Chebyshev and Laguerre polynomials
FL product of Fibonacci and Laguerre polynomials
LLE product of Legendre and Laguerre polynomials

Many analytical and numerical methods are developed to solve different types of FVIEs. Adomain Decomposition, Quadrature, Galerkin, Collocation, different version of Wavelet, successive substitutions methods are introduced and researched by many researchers. Maleknejad and Hadizadeh [1] suggested Adomain decomposition method for mixed nonlinear FVIE and they also discussed about the limitof it. Maleknejad and FadaeiYami [2] used Adomain decomposition method to solve the system of VFIEs. Hendi and Bakodah [3] compared approximate solution with the exact solution which is obtained from discrete Adomain decomposition method. They used FVIE in two dimensional space and reported that it's a method that can be used to solve nonlinear integral equations in two dimensional space. Hendi and Albugami [4] solved FVIE of second kind using Galerkin and Collocation method and they considered continuous kernel with respect to position and time. Moreover, Mustafa and Ghanim [5] discussed quadrature methods i.e, Trapezoidal rule, Weddle's rule and Richardson's extrapolation to solve linear VFIEs numerically. More recently, Molla and Saha [6]showed the approximate solution of FVIE of second kind using the methods used by Hendi and Albugami [4]. They have reported that the performance of different polynomials and collocation points are consistent for both these methods. Abdou et al. [7] established the general solution of VFIEs with discontinuous kernel in a Banach Space using separation of variables method.

In this research, Galerkin method is used to find the approximate solution of FVIE of 2nd kind where new set of polynomials (BF, CH, CL, FL, LLE polynomials) are used as basis functions. Here, BF refers to product of Bernstein and Fibonacci polynomials, $\mathrm{CH}$ refers to product of Chebyshev and Hermite polynomials, CL refers to product of Chebyshev and Laguerre polynomials, FL refers to product of Fibonacci and Laguerre polynomials, LLE refers to product of Legendre and Laguerre polynomials. As far our knowledge, BF, CH, CL, FL, LLE polynomials are not introduced before to find the approximate solutions of FVIE of second kind. In recent times, Molla and Saha [8] used LH polynomials as basis function in Galerkin method for finding approximate solution of FIE of 2nd kind within a very short description. A brief discussion about the performance of above mentioned polynomials for the solution of FVIE of 2nd kind is presented in this paper.

This article is designed as follows: In §2, brief introduction of five new set of polynomials (BF, CH, CL, FL, LLE) are given. After that, details formulation of Galerkin method in order to find approximate solutions of FVIE of 2nd kind is illustrated in $\S 3$. Then, numerical results of the Galerkin method using different polynomials are computed, consequent absolute errors are presented graphically and RMSE and MAE are calculated in $\S 4$. Finally, the study concludes with a brief conclusion in $\S 5$.

\section{Introduction of Polynomials}

Hermite, Chebyshev, Laguerre, Fibonacci and Legendre polynomials are the most widely used classical orthogonal polynomials in approximation theory and numerical analysis and they used often in order to find the approximate solutions of different kinds of integral equations. In this research, some special and new sets of polynomials are introduced, and they are obtained as a product of the well known polynomials. Product of Bernstein and Fibonacci polynomials (BF):

$$
B F_{i}(u)=B_{i}(u) F_{i}(u)
$$

Here we use fourth degree Bernstein polynomials over the interval $[0,1]$ and first five BF polynomials are presented underneath: 


$$
\left.\begin{array}{c}
B F_{0}(u)=1-4 u+6 u^{2}-4 u^{3}+u^{4} \\
B F_{1}(u)=4 u^{2}-12 u^{3}+12 u^{4}-4 u^{5} \\
B F_{2}(u)=6 u^{2}-12 u^{3}+12 u^{4}-12 u^{5}+6 u^{6} \\
B F_{3}(u)=8 u^{4}-8 u^{5}+4 u^{6}-4 u^{7} \\
B F_{4}(u)=u^{4}+3 u^{6}+u^{8}
\end{array}\right\}
$$

Product of Chebyshev and Hermite polynomials $(\mathrm{CH})$ :

$$
\mathrm{CH}_{i}(u)=C_{i}(u) H_{i}(u)
$$

Also, some of the $\mathrm{CH}$ polynomials are shown here:

$$
\left.\begin{array}{c}
\mathrm{CH}_{0}(u)=1 \\
\mathrm{CH}_{1}(u)=2 u^{2} \\
\mathrm{CH}_{2}(u)=2-8 u^{2}+8 u^{4} \\
\mathrm{CH}_{3}(u)=36 u^{2}-72 u^{4}+32 u^{6} \\
\mathrm{CH}_{4}(u)=12-144 u^{2}+496 u^{4}-512 u^{6}+128 u^{8}
\end{array}\right\}
$$

Product of Chebyshev and Laguerre polynomials (CL):

$$
C L_{i}(u)=C_{i}(u) L_{i}(u)
$$

Also, some of the CL polynomials are shown here:

$$
\left.\begin{array}{c}
C L_{0}(u)=1 \\
C L_{1}(u)=u-u^{2} \\
C L_{2}(u)=-1+2 u+\frac{3 u^{2}}{2}-4 u^{3}+u^{4} \\
C L_{3}(u)=-3 u+9 u^{2}-\frac{u^{3}}{2}-\frac{23 u^{4}}{2}+6 u^{5}-\frac{2 u^{6}}{3} \\
C L_{4}(u)=1-4 u-5 u^{2}+\frac{94 u^{3}}{3}-\frac{383 u^{4}}{24}-\frac{80 u^{5}}{3}+\frac{71 u^{6}}{3}-\frac{16 u^{7}}{3}+\frac{u^{8}}{3}
\end{array}\right\}
$$

Product of Fibonacci and Laguerre polynomials (FL):

$$
F L_{i}(u)=F_{i}(u) L_{i}(u)
$$

Also, some of the FL polynomials are shown here:

$$
\left.\begin{array}{c}
F L_{0}(u)=1 \\
F L_{1}(u)=u-u^{2} \\
F L_{2}(u)=1-2 u+\frac{3 u^{2}}{2}-2 u^{3}+\frac{u^{4}}{2} \\
F L_{3}(u)=2 u-6 u^{2}+4 u^{3}-\frac{10 u^{4}}{3}+\frac{3 u^{5}}{2}-\frac{u^{6}}{6} \\
F L_{4}(u)=1-4 u+6 u^{2}-\frac{38 u^{3}}{3}+\frac{241 u^{4}}{24}-6 u^{5}+\frac{25 u^{6}}{8}-\frac{2 u^{7}}{3}+\frac{u^{8}}{24}
\end{array}\right\}
$$

Product of Laguerre and Legendre polynomials (LLE):

$$
L L E_{i}(u)=L_{i}(u) L E_{i}(u)
$$

Also, some of the LLE polynomials are shown here: 


$$
\left.\begin{array}{c}
L L E_{0}(u)=1 \\
L L E_{1}(u)=u-u^{2} \\
L L E_{2}(u)=-\frac{1}{2}+u+\frac{5 u^{2}}{4}-3 u^{3}+\frac{3 u^{4}}{4} \\
L L E_{3}(u)=-\frac{3 u}{2}+\frac{9 u^{2}}{2}+\frac{u^{3}}{4}-\frac{29 u^{4}}{4}+\frac{15 u^{5}}{4}-\frac{5 u^{6}}{12} \\
L L E_{4}(u)=\frac{3}{8}-\frac{3 u}{2}-\frac{21 u^{2}}{8}+\frac{59 u^{3}}{4}-\frac{439 u^{4}}{64}-15 u^{5}+\frac{415 u^{6}}{32}-\frac{35 u^{7}}{12}+\frac{35 u^{8}}{192}
\end{array}\right\}
$$

\section{Numerical Method}

In Galerkin methods [9], solution is obtained in terms of basis functions. In order to find the approximate solution, next step is to determine the unknown parameters in trial solution. So Galerkin method gives a polynomial as an approximate solution at each points instead of values, and we can find values at any points within the defined domain. The details of the numerical formulation of FVIE of 2nd kind are presented in [6].

\section{Results and Discussion}

Galerkin method is used to obtain the approximate solution of linear FVIE of 2nd kind, and we tried to explore the performance of Galerkin method by using a set of new types of polynomials (BF, CH, CL, FL, LLE polynomials). Here, we use exact solution of the numerical problems in order to check the accuracy of our code and to examine the recital of different polynomials with regard to absolute errors. Numerical results and graphs are generated by using Wolfram Mathematica 9 .

In the first section, we will investigate the approximate solutions of the problem using Galerkin method where we will use BF, CH, CL, FL, LLE polynomials as basis functions. Secondly, we will see the performance of basis functions by observing error graphs using $n=2,3,4$, 5. Finally, we will calculate RMSE and MAE for the numerical solutions to observe the accuracy of Galerkin method for different basis functions. Here we consider two numerical examples to carry out the investigations and in both examples, domain of the problem is $[0,1]$.

Example 1: [6]

$$
\phi(x)+\int e^{x+t} \phi(t) d t-\int e^{x+t} \phi(t) d t=e^{-x}-(x-1) e^{x}, 0 \leq x \leq 1
$$

Exact solution of $\phi(x)$ is $\phi(x)=e^{-x}$.

Firstly, Galerkin solution for linear FVIE of second kind with $n=2,3,4,5$ are presented in Tables A1 to A4 with five special types of polynomials (BF, CH, CL, FL, LLE) called as basis functions. From Tables A1 to A4, it is evident that using BF, CH, CL, FL, LLE polynomials as basis function in Galerkin method in order to find numerical approximations of FVIEs of $2^{\text {nd }}$ kind are possible, and it has shown that numerical approximations of all the polynomials are converging into the same direction. It is also examined that polynomial solutions in Galerkin method using BF, CH, CL, FL, LLE polynomials are different, but approximate solutions are approximately same for each of these polynomials with same degree. For example, in Table A5 we have shown five approximate polynomials solution using five different polynomials as basis function for $\mathrm{n}=5$ to verify the previous statement. It suggests that introducing these polynomials can be functional and these polynomials can be used to obtain approximate solutions in Galerkin method. And it is seen that there is an insignificant effect on the solution of FVIEs compared with the exact solution. In the second part, from Fig. 4.1, we have observed absolute error graphs of five new polynomials over the interval $[0,1]$ for $n=2,3,4,5$. 


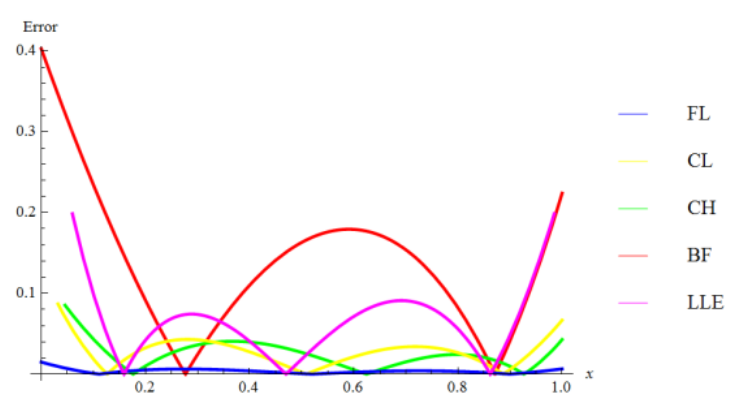

$n=2$

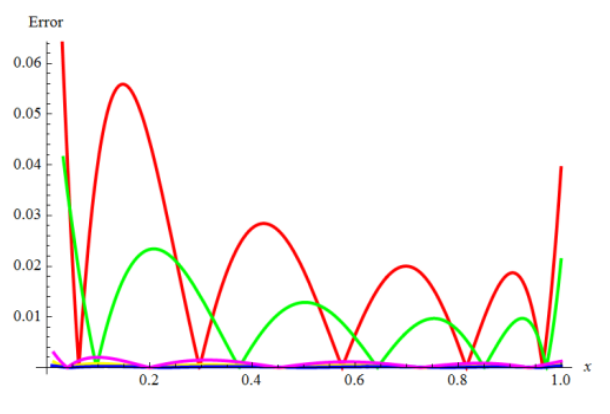

$n=4$

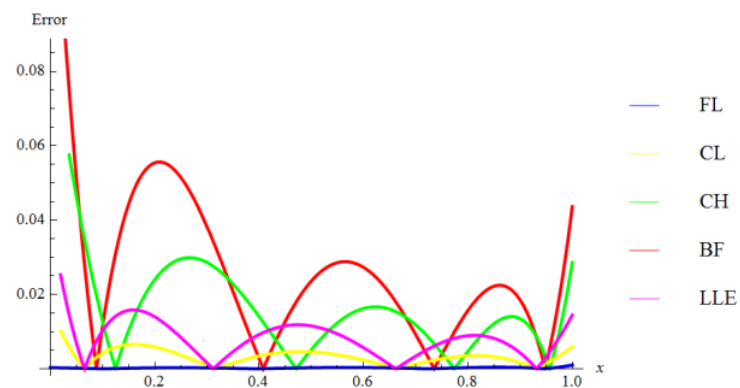

$n=3$

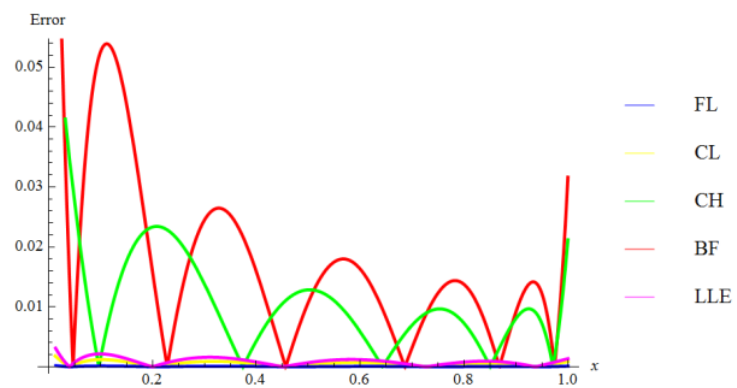

$n=5$

Fig. 4.1: Absolute error graph for Example 1

From this observation, we can claim that errors are decreasing as the values of $\mathrm{n}$ are increased. Hence, by increasing the values of $\mathrm{n}$ we can get desired accuracy using those basis polynomials. For this example, absolute errors are relatively high for $\mathrm{BF}$ and $\mathrm{CH}$ polynomials compared to the other polynomials. On the other hand, for FL polynomial absolute errors are significantly small for each values of $\mathrm{n}$ compared to the other polynomials.

Finally, we want to justify and understand our approximate solutions and error graphs more precisely and for this reason standard errors such as Mean Absolute Error (MAE) and Root Mean Square Error (RMSE) are observed for the approximate solutions. RMSE and MAE are given in Table 4.1 for different values of $n$ with five different polynomials.

Table 4.1: RMSE and MAE results for Example 1

\begin{tabular}{c|c|c|c|c}
\hline & \multicolumn{2}{|c|}{$\mathrm{n}=2$} & \multicolumn{2}{c}{$\mathrm{n}=3$} \\
\hline Polynomials & RMSE & MAE & RMSE & MAE \\
\hline BF & 0.1856936 & 0.1551874 & 0.0534938 & 0.0361026 \\
\hline CH & 0.0472953 & 0.0346472 & 0.0321639 & 0.0227020 \\
\hline CL & 0.0501415 & 0.0370874 & 0.0061832 & 0.00458443 \\
\hline FL & 0.0059065 & 0.0044154 & 0.0003886 & 0.00029313 \\
\hline LLE & 0.1513725 & 0.1066698 & 0.0152785 & 0.01132040 \\
\hline \multicolumn{2}{|c|}{$\mathrm{n}=4$} & \multicolumn{2}{c}{$\mathrm{n}=5$} \\
\hline Polynomials & RMSE & MAE & RMSE & MAE \\
\hline BF & 0.0532509 & 0.0343142 & 0.0511507 & 0.0314623 \\
\hline CH & 0.0242799 & 0.0159440 & 0.0242745 & 0.0159268 \\
\hline CL & 0.0007080 & 0.0004955 & 0.0010927 & 0.0007841 \\
\hline FL & 0.0002347 & 0.0001849 & 0.0001209 & 0.0000852 \\
\hline LLE & 0.0018356 & 0.0012656 & 0.0019451 & 0.0013528 \\
\hline
\end{tabular}


Above information shows that in all cases, both RMSEs and MAEs are decreased by increasing the values of $\mathrm{n}$. It is again evident that FL polynomial gives the best accuracy of the approximate solution among the other polynomials for each values of $n=2,3,4,5$. Thus, for FL polynomial both errors are significantly small.

Example 2: [6]

where

$$
\phi(x)+\int e^{t} \sin x \phi(t) d t-\int e^{t} \cos x \phi(t) d t=f(x), 0 \leq x \leq 1
$$

$$
f(x)=e^{x}-\frac{1}{2}\left(e^{2 x}-1\right) \cos x+\frac{1}{2}\left(e^{2}-1\right) \sin x
$$

Exact solution of $\phi(x)$ is $\phi(x)=e^{x}$

Similar procedures have also done for the above problem, and try to carry out our observation for this numerical example. Results are presented in Tables A6 to A9 with n = 2, 3, 4, 5. From Tables A6 to A9, It is again remarked that we can find numerical solution of FVIE of 2nd by using BF, CH, CL, FL, LLE polynomials as basis function in Galerkin method, and we have got significant result for each case. Secondly, we observe absolute error graphs for BF, CH, CL, FL, LLE polynomials in Galerkin method over the interval $[0,1]$ which are presented in Fig. 4.2.
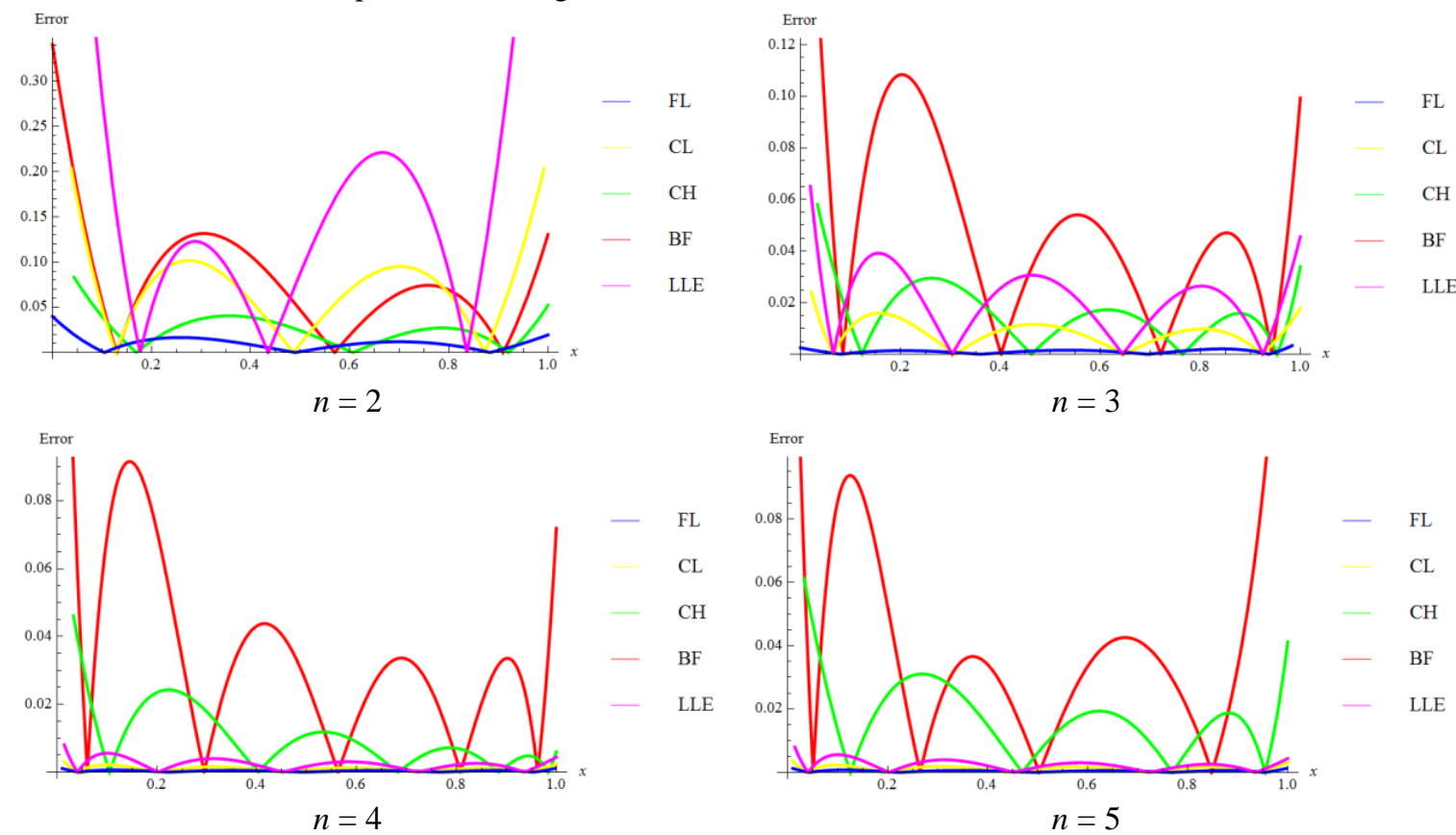

Fig.4.2: Absolute error graph for Example 2

We observe that errors reduce gradually as the values of $n$ increase. For $n=4 \& 5$, absolute errors graphs are little high for $\mathrm{BF}$ and $\mathrm{CH}$ polynomials compared to the other polynomials. Similar findings are also observed for Example 1. It is observed that $\mathrm{BF}$ and $\mathrm{CH}$ polynomials exhibit higher functional values compare to other polynomials. This may play an important role to show higher absolute errors for these polynomials. According to the observation of the absolute error graphs, FL polynomial gives comparatively better results for each values of $n$ in order to get approximate solutions of FVIEs of second kind in Galerkin method. In the last part, we have observed RMSE and MAE for each approximate solutions. RMSE and MAE are presented in Table 4.2 for $n=2,3,4$ and 5 .

Table 4.2: RMSE and MAE results for Example 2

\begin{tabular}{c|c|c|c|c}
\hline & \multicolumn{2}{|c|}{$\mathrm{n}=2$} & \multicolumn{2}{c}{$\mathrm{n}=3$} \\
\hline Polynomials & RMSE & MAE & RMSE & MAE \\
\hline BF & 0.129489 & 0.0967744 & 0.1033868 & 0.0704576 \\
\hline CH & 0.047037 & 0.0349461 & 0.0322473 & 0.0232427 \\
\hline
\end{tabular}




\begin{tabular}{c|c|c|c|c}
\hline CL & 0.134701 & 0.1010860 & 0.0157194 & 0.0116686 \\
\hline FL & 0.015932 & 0.0119732 & 0.0019901 & 0.0015194 \\
\hline LLE & 0.399733 & 0.2743080 & 0.0401397 & 0.0298389 \\
\hline \multicolumn{2}{|c|}{$\mathrm{n}=4$} & \multicolumn{2}{c}{$\mathrm{n}=5$} \\
\hline Polynomials & RMSE & MAE & RMSE & MAE \\
\hline BF & 0.0866670 & 0.056482 & 0.1027431 & 0.0695213 \\
\hline CH & 0.0250143 & 0.014217 & 0.0342948 & 0.0253551 \\
\hline CL & 0.0020563 & 0.001499 & 0.0022762 & 0.0017664 \\
\hline FL & 0.0006835 & 0.000535 & 0.0008029 & 0.0006282 \\
\hline LLE & 0.0052145 & 0.003625 & 0.0052129 & 0.0036167 \\
\hline
\end{tabular}

Based on above information, in all cases both RMSEs and MAEs are decreased by increasing the values of n. So, same conclusion is also considerable for this problem.

Example 3:

where

$$
\phi(x)+\int_{0}^{1} e^{x-t} \phi(t) d t-\int_{0}^{x}(x-t) \phi(t) d t=f(x), 0 \leq x \leq 1
$$

$$
f(x)=e^{x}+x+1
$$

Exact solution of $\phi(x)$ is $\phi(x)=e^{x}$.

Accordingly, Tables A11 to A14 showed approximate solutions of linear FVIE of $2^{\text {nd }}$ kind using Galerkin method for $n=2,3,4,5$ with BF, CH, CL, FL and LLE polynomials as basis functions. In Table A15, we show approximate polynomial solution for BF, CH, CL, FL, LLE polynomials (using as basis function in Galerkin method) with $n=5$, and each polynomial is a polynomial of degree 10 . Then, we extent our observation by representing error graphs for BF, CH, CL, FL, LLE polynomials in Galerkin method over the interval $[0,1]$ and they are presented in Fig. 4.3.
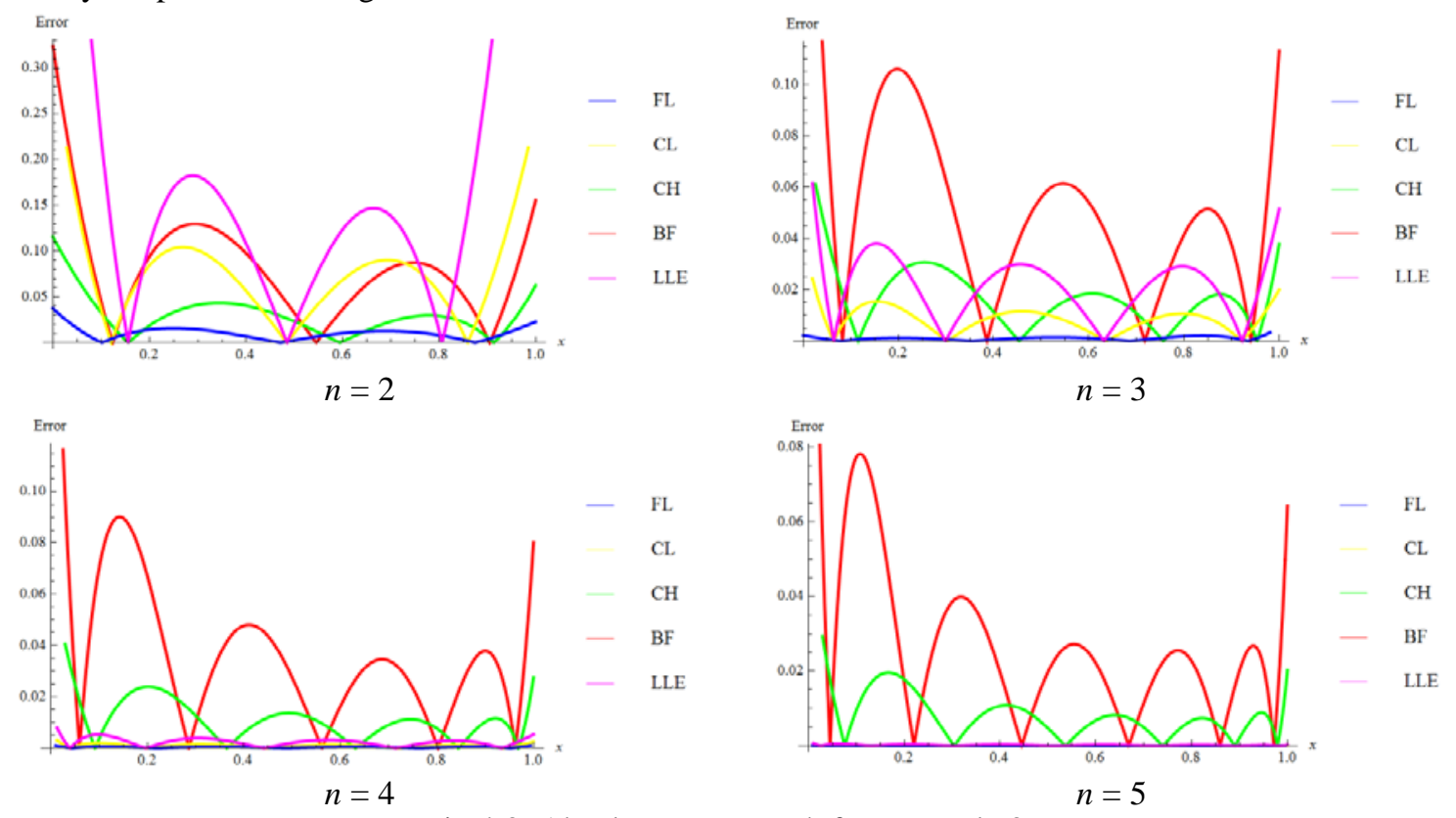

Fig.4.3: Absolute error graph for Example 3

We examine that absolute errors are decreasing significantly as the values of $n$ increase. So, by increasing the values of n, it is possible to obtain desire accuracy of the problem. Finally, we have observed RMSE and MAE for each approximate solutions, and they are presented in Table 4.3 for $n=2,3,4$ and 5 . 
Table 4.3: RMSE and MAE results for Example 3

\begin{tabular}{c|c|c|c|c}
\hline & \multicolumn{2}{|c|}{$\mathrm{n}=2$} & \multicolumn{2}{c}{$\mathrm{n}=3$} \\
\hline Polynomials & RMSE & MAE & RMSE & MAE \\
\hline BF & 0.128485 & 0.0981224 & 0.1028080 & 0.0729980 \\
\hline CH & 0.046646 & 0.0357625 & 0.0319543 & 0.0237026 \\
\hline CL & 0.133949 & 0.1011620 & 0.0155413 & 0.0116668 \\
\hline FL & 0.015758 & 0.0121843 & 0.0020232 & 0.0015067 \\
\hline LLE & 0.394175 & 0.265560 & 0.0396560 & 0.0298728 \\
\hline \multicolumn{2}{|c|}{$\mathrm{n}=4$} & \multicolumn{2}{c}{$\mathrm{n}=5$} \\
\hline Polynomials & RMSE & MAE & RMSE & MAE \\
\hline BF & 0.0861604 & 0.0578768 & 0.0748337 & 0.0482383 \\
\hline CH & 0.0242705 & 0.0169154 & 0.0193992 & 0.0126937 \\
\hline CL & 0.0020327 & 0.0014985 & 0.0001662 & 0.0001134 \\
\hline FL & 0.0006825 & 0.0005388 & 0.0000536 & 0.0000413 \\
\hline LLE & 0.0050217 & 0.0036309 & 0.0005045 & 0.0003298 \\
\hline
\end{tabular}

According to the above information, in all cases both RMSEs and MAEs are decreased by increasing the values of n, and FL polynomial gives better result.

\section{Conclusion}

In this paper, new polynomials called BF, CH, CL, FL, LLE polynomials are introduced, and among them $\mathrm{CH}, \mathrm{CL}, \mathrm{FL}$ and LLE polynomials are polynomials of degree 2n. Then Linear FVIE of second kind is solved by Galerkin method where BF, CH, CL, FL, LLE polynomials are considered as basis function. Moreover, RMSE and MAE are calculated for each approximate solutions to examine the performance of basis functions in Galerkin method. And it is seen that in Galerkin method all approximate solutions are almost similar for different basis functions with same power. It is also seen that BF, FL, LLE and CL polynomials take less time than BF polynomials. Throughout this observation, we claim that it is possible to find approximate solutions of FVIE of 2nd kind by using BF, CH, CL, FL, LLE polynomials as basis function in Galerkin method. We have also examined that FL polynomial which is considered as basis function in Galerkin method gives us better result compared to the other basis functions (BF, CH, CL, LLE polynomials).

Conflict of Interest

The authors do not report any financial or personal connections with other persons or organizations, which might negatively affect the contents of this publication and/or claim authorship rights to this publication.

Publication Ethics

Submitted manuscripts must not have been previously published by or be under review by another print or online journal or source.

Funding

This research work is a self funded research. 


\section{References}

[1] Maleknejad, K. \& Hadizadeh, M. (1999). A new computational method for Volterra-Fredholm integral equation. Computers and Mathematics with Applications, 37, 1-8.

[2] Maleknejad, K. \& FadaeiYami, M.R. (2006). A computational method for system of VolterraFredholm integral equations. Applied Mathematics and Computation, 183(1),589-595.

[3] Hendi, F.A. \& Bakodah, H.O. (2012). Numerical solution of Fredholm-Volterra integral equation in two dimensional space by using discrete Adomain decomposition method. IJRRAS, 10(3), 466-471.

[4] Hendi, F.A. \& Albugami, A.M. (2009). Numerical solution for Fredholm-Volterra integral equation of the second kind by using collocation and Galerkin methods. Journal of King Saud University (Science), 22, 37-40.doi: 10.1016/j. jksus. 2009. 12. 006

[5] Mustafa, M.M. \& Ghanim, I.N. (2018). Numerical solution of linear Volterra-Fredholm integral equations using quadrature methods.

[6] Molla, H.U. \& Saha, G. (2020). Numerical approximations of Fredholm-Volterra integral equation of second kind using Galerkin and Collocation methods. Suan Sunandha Science and Technology Journal, 7(2), 15-22.

[7] Abdou, M.A., Soliman, A.A. \& Abdel-Aty, M.A. (2020). On a discussion of Volterra-Fredholm integral equation with discontinuous kernel. Journal of the Egyptian Mathematical Society, 28, 1-10.

[8] Molla, H.U. \& Saha, G. (2018). Numerical approximation of Fredholm integral equation (FIE) of 2nd kind using Galerkin and collocation methods. GANIT: Journal of Bangladesh Mathematical Society, 38,11-25.doi:10.3329/ganit.v38i0.39782

[9] Lewis, P. E. \& Ward, H. R. (1991). The finite element method: Principles and application. AddisonWesley Publishing Company. 
APPENDIX A:

Table A1: Numerical results for Example $1(n=2)$

\begin{tabular}{c|c|c|c|c|c|c}
\hline$x$ & EXACT & BF & CH & CL & FL & LLE \\
\hline 0.0 & 1.000000 & 1.402747 & 0.872596 & 0.870121 & 1.014979 & 0.599279 \\
\hline 0.1 & 0.904837 & 1.139234 & 0.861446 & 0.886132 & 0.905866 & 0.805545 \\
\hline 0.2 & 0.818731 & 0.910140 & 0.828788 & 0.851282 & 0.813534 & 0.860804 \\
\hline 0.3 & 0.740818 & 0.716518 & 0.776997 & 0.783340 & 0.734651 & 0.814721 \\
\hline 0.4 & 0.670320 & 0.560129 & 0.710031 & 0.697982 & 0.666275 & 0.711115 \\
\hline 0.5 & 0.606531 & 0.443447 & 0.633432 & 0.608796 & 0.605857 & 0.587965 \\
\hline 0.6 & 0.548812 & 0.369653 & 0.554324 & 0.527277 & 0.551241 & 0.477405 \\
\hline 0.7 & 0.496585 & 0.342642 & 0.481415 & 0.462832 & 0.500662 & 0.405729 \\
\hline 0.8 & 0.449329 & 0.367015 & 0.424997 & 0.422777 & 0.452746 & 0.393385 \\
\hline 0.9 & 0.406569 & 0.448087 & 0.396944 & 0.412337 & 0.406515 & 0.454980 \\
\hline 1.0 & 0.367879 & 0.591881 & 0.410714 & 0.434648 & 0.361379 & 0.599279 \\
\hline
\end{tabular}

Table A2: Numerical results for Example $1(n=3)$

\begin{tabular}{c|c|c|c|c|c|c}
\hline$x$ & EXACT & BF & CH & CL & FL & LLE \\
\hline 0.0 & 1.000000 & 1.151427 & 0.909564 & 0.983350 & 1.000283 & 0.958811 \\
\hline 0.1 & 0.904837 & 0.893436 & 0.892129 & 0.909014 & 0.904846 & 0.915063 \\
\hline 0.2 & 0.818731 & 0.763348 & 0.842693 & 0.824616 & 0.818468 & 0.832715 \\
\hline 0.3 & 0.740818 & 0.702516 & 0.769416 & 0.741811 & 0.740574 & 0.742461 \\
\hline 0.4 & 0.670320 & 0.667598 & 0.684387 & 0.666867 & 0.670334 & 0.661109 \\
\hline 0.5 & 0.606531 & 0.630116 & 0.601363 & 0.601975 & 0.606820 & 0.594997 \\
\hline 0.6 & 0.548812 & 0.576096 & 0.532601 & 0.546445 & 0.549162 & 0.543118 \\
\hline 0.7 & 0.496585 & 0.505819 & 0.484781 & 0.497807 & 0.496679 & 0.499986 \\
\hline 0.8 & 0.449329 & 0.433663 & 0.454034 & 0.452799 & 0.449009 & 0.458221 \\
\hline 0.9 & 0.406569 & 0.388805 & 0.420051 & 0.408261 & 0.406215 & 0.410863 \\
\hline 1.0 & 0.367879 & 0.411482 & 0.339298 & 0.361912 & 0.368884 & 0.353421 \\
\hline
\end{tabular}

Table A3: Numerical results for Example $1(n=4)$

\begin{tabular}{c|c|c|c|c|c|c}
\hline$x$ & EXACT & BF & CH & CL & FL & LLE \\
\hline 0.0 & 1.000000 & 1.155063 & 0.929939 & 0.998042 & 0.999456 & 0.994885 \\
\hline 0.1 & 0.904837 & 0.863940 & 0.906318 & 0.905615 & 0.905061 & 0.906862 \\
\hline 0.2 & 0.818731 & 0.774149 & 0.842058 & 0.818743 & 0.818807 & 0.818722 \\
\hline 0.3 & 0.740818 & 0.742091 & 0.754639 & 0.740257 & 0.740667 & 0.739346 \\
\hline 0.4 & 0.670320 & 0.697897 & 0.665973 & 0.670017 & 0.670151 & 0.669564 \\
\hline 0.5 & 0.606531 & 0.625539 & 0.593669 & 0.606764 & 0.606519 & 0.607169 \\
\hline 0.6 & 0.548812 & 0.542236 & 0.542974 & 0.549244 & 0.548956 & 0.549918 \\
\hline 0.7 & 0.496585 & 0.476581 & 0.503573 & 0.496725 & 0.496724 & 0.496899 \\
\hline 0.8 & 0.449329 & 0.444874 & 0.456606 & 0.449053 & 0.449284 & 0.448609 \\
\hline 0.9 & 0.406569 & 0.425150 & 0.398469 & 0.406342 & 0.406405 & 0.406042 \\
\hline 1.0 & 0.367879 & 0.328439 & 0.389164 & 0.368410 & 0.368244 & 0.369117 \\
\hline
\end{tabular}


Table A4: Numerical results for Example $1(n=5)$

\begin{tabular}{c|c|c|c|c|c|c}
\hline$x$ & EXACT & BF & CH & CL & FL & LLE \\
\hline 0.0 & 1.000000 & 1.152473 & 0.929920 & 0.997018 & 1.000333 & 0.994609 \\
\hline 0.1 & 0.904837 & 0.852263 & 0.906303 & 0.906036 & 0.904706 & 0.906984 \\
\hline 0.2 & 0.818731 & 0.802881 & 0.842055 & 0.818795 & 0.818717 & 0.818717 \\
\hline 0.3 & 0.740818 & 0.765297 & 0.756515 & 0.739939 & 0.740905 & 0.739239 \\
\hline 0.4 & 0.670320 & 0.686192 & 0.665995 & 0.669803 & 0.670383 & 0.669510 \\
\hline 0.5 & 0.606531 & 0.595780 & 0.593684 & 0.606879 & 0.606513 & 0.607225 \\
\hline 0.6 & 0.548812 & 0.532499 & 0.542967 & 0.549516 & 0.548746 & 0.550011 \\
\hline 0.7 & 0.496585 & 0.499803 & 0.503546 & 0.496832 & 0.496545 & 0.496924 \\
\hline 0.8 & 0.449329 & 0.463076 & 0.456593 & 0.448883 & 0.449357 & 0.448541 \\
\hline 0.9 & 0.406569 & 0.397390 & 0.398506 & 0.406191 & 0.406619 & 0.405995 \\
\hline 1.0 & 0.367879 & 0.399509 & 0.389068 & 0.368743 & 0.367772 & 0.369225
\end{tabular}

Table A5: Approximate polynomial solutions for Example $1(n=5)$

\begin{tabular}{|c|c|}
\hline Polynomials & Approximate polynomial solutions $(\tilde{\varphi}(x))$ \\
\hline $\mathrm{BF}$ & $\begin{array}{l}1.15247345-5.7623672 x+38.190357 x^{2}-123.676961 x^{3}+191.28067 x^{4}-136.574009 x^{5} \\
+38.150331 x^{6}-9.717654 x^{7}+7.637876 x^{8}-0.33114693 x^{9} \\
+0.049938671 x^{10}\end{array}$ \\
\hline $\mathrm{CH}$ & $0.92992-2.4195 x^{2}+5.8451 x^{4}-6.9284 x^{6}+2.9674 x^{8}-0.0054445 x^{10}$ \\
\hline CL & $\begin{array}{c}0.99702-0.89614 x-0.372667 x^{2}+2.79001 x^{3}-4.6447 x^{4}+3.31052 x^{5}-0.47132 x^{6} \\
-0.55406 x^{7}+0.240349 x^{8}-0.031513 x^{9}+0.00126052 x^{10}\end{array}$ \\
\hline FL & $\begin{array}{l}1.000333-1.011737 x+0.60256 x^{2}-0.550671 x^{3}+0.800712 x^{4}-0.890065 x^{5} \\
+0.640786 x^{6}-0.292145 x^{7}+0.0767605 x^{8}-0.00912658 x^{9} \\
+0.000365063 x^{10}\end{array}$ \\
\hline LLE & $\begin{array}{c}0.9946-0.8062 x-1.1962 x^{2}+5.9365 x^{3}-10.692 x^{4}+9.587 x^{5}-4.0378 x^{6}+0.55248 x^{7} \\
+0.041717 x^{8}-0.011685 x^{9}+0.00046741 x^{10}\end{array}$ \\
\hline
\end{tabular}

Table A6: Numerical results for Example $2(n=2)$

\begin{tabular}{c|c|c|c|c|c|c}
\hline$x$ & EXACT & BF & CH & CL & FL & LLE \\
\hline 0.0 & 1.000000 & 1.340495 & 1.123314 & 1.330865 & 0.960178 & 1.994119 \\
\hline 0.1 & 1.105171 & 1.160955 & 1.144839 & 1.153858 & 1.103909 & 1.387584 \\
\hline 0.2 & 1.221403 & 1.132489 & 1.208674 & 1.142888 & 1.236002 & 1.172719 \\
\hline 0.3 & 1.349859 & 1.218369 & 1.312602 & 1.250363 & 1.365524 & 1.228219 \\
\hline 0.4 & 1.491825 & 1.384966 & 1.452928 & 1.434292 & 1.500473 & 1.447048 \\
\hline 0.5 & 1.648721 & 1.601759 & 1.624478 & 1.658282 & 1.647782 & 1.736440 \\
\hline 0.6 & 1.822119 & 1.841333 & 1.820598 & 1.891538 & 1.813315 & 2.017904 \\
\hline 0.7 & 2.013753 & 2.079377 & 2.033160 & 2.108865 & 2.001874 & 2.227218 \\
\hline 0.8 & 2.225541 & 2.294685 & 2.252554 & 2.290668 & 2.217189 & 2.314432 \\
\hline 0.9 & 2.459603 & 2.469158 & 2.467693 & 2.422948 & 2.461928 & 2.243868 \\
\hline 1.0 & 2.718282 & 2.587802 & 2.666012 & 2.497308 & 2.737691 & 1.994119 \\
\hline
\end{tabular}


Table A7: Numerical results for Example $2(n=3)$

\begin{tabular}{c|c|c|c|c|c|c}
\hline$x$ & EXACT & BF & CH & CL & FL & LLE \\
\hline 0.0 & 1.000000 & 1.287770 & 1.088912 & 1.041651 & 0.997498 & 1.106021 \\
\hline 0.1 & 1.105171 & 1.075753 & 1.116687 & 1.094703 & 1.105588 & 1.079326 \\
\hline 0.2 & 1.221403 & 1.113039 & 1.197124 & 1.207495 & 1.228030 & 1.187712 \\
\hline 0.3 & 1.349859 & 1.279609 & 1.322029 & 1.348566 & 1.350633 & 1.348632 \\
\hline 0.4 & 1.491825 & 1.491080 & 1.479356 & 1.501197 & 1.491285 & 1.517373 \\
\hline 0.5 & 1.648721 & 1.695569 & 1.655607 & 1.659813 & 1.647235 & 1.677731 \\
\hline 0.6 & 1.822119 & 1.871219 & 1.839203 & 1.826688 & 1.820821 & 1.833428 \\
\hline 0.7 & 2.013753 & 2.024405 & 2.024819 & 2.008925 & 2.013859 & 2.000284 \\
\hline 0.8 & 2.225541 & 2.188612 & 2.218677 & 2.215728 & 2.227315 & 2.199126 \\
\hline 0.9 & 2.459603 & 2.420016 & 2.444802 & 2.455963 & 2.460987 & 2.449448 \\
\hline 1.0 & 2.718282 & 2.817638 & 2.752245 & 2.736002 & 2.713251 & 2.763819 \\
\hline
\end{tabular}

Table A8: Numerical results for Example $2(n=4)$

\begin{tabular}{c|c|c|c|c|c|c}
\hline$x$ & EXACT & BF & CH & CL & FL & LLE \\
\hline 0.0 & 1.000000 & 1.250063 & 1.075603 & 1.005443 & 1.001539 & 1.014459 \\
\hline 0.1 & 1.105171 & 1.035707 & 1.107548 & 1.103017 & 1.104544 & 1.099588 \\
\hline 0.2 & 1.221403 & 1.150292 & 1.197907 & 1.221340 & 1.221217 & 1.221227 \\
\hline 0.3 & 1.349859 & 1.353454 & 1.332069 & 1.351411 & 1.350290 & 1.353777 \\
\hline 0.4 & 1.491825 & 1.534912 & 1.491309 & 1.492666 & 1.492259 & 1.493902 \\
\hline 0.5 & 1.648721 & 1.674947 & 1.659734 & 1.648026 & 1.648696 & 1.647018 \\
\hline 0.6 & 1.822119 & 1.806986 & 1.831007 & 1.820838 & 1.821687 & 1.819122 \\
\hline 0.7 & 2.013753 & 1.980388 & 2.011856 & 2.013361 & 2.013400 & 2.012971 \\
\hline 0.8 & 2.225541 & 2.221737 & 2.218509 & 2.226495 & 2.225741 & 2.227727 \\
\hline 0.9 & 2.459603 & 2.493157 & 2.461356 & 2.460435 & 2.460091 & 2.461194 \\
\hline 1.0 & 2.718282 & 2.646382 & 2.712265 & 2.715999 & 2.717108 & 2.713881 \\
\hline
\end{tabular}

Table A9: Numerical results for Example $2(n=5)$

\begin{tabular}{c|c|c|c|c|c|c}
\hline$x$ & EXACT & BF & CH & CL & FL & LLE \\
\hline 0.0 & 1.000000 & 1.254249 & 1.091654 & 1.005527 & 1.001895 & 1.014482 \\
\hline 0.1 & 1.105171 & 1.019803 & 1.118548 & 1.102837 & 1.104430 & 1.099590 \\
\hline 0.2 & 1.221403 & 1.168899 & 1.196909 & 1.221612 & 1.221193 & 1.221206 \\
\hline 0.3 & 1.349859 & 1.370233 & 1.319937 & 1.351750 & 1.350369 & 1.353762 \\
\hline 0.4 & 1.491825 & 1.525867 & 1.476966 & 1.492646 & 1.492329 & 1.493911 \\
\hline 0.5 & 1.648721 & 1.650158 & 1.654892 & 1.647643 & 1.648683 & 1.647044 \\
\hline 0.6 & 1.822119 & 1.788651 & 1.840925 & 1.820464 & 1.821612 & 1.819141 \\
\hline 0.7 & 2.013753 & 1.972185 & 2.027304 & 2.013387 & 2.013346 & 2.012965 \\
\hline 0.8 & 2.225541 & 2.205479 & 2.218559 & 2.226918 & 2.225780 & 2.227700 \\
\hline 0.9 & 2.459603 & 2.492856 & 2.441719 & 2.460673 & 2.460170 & 2.461182 \\
\hline 1.0 & 2.718282 & 2.906693 & 2.759489 & 2.715178 & 2.716914 & 2.713927 \\
\hline
\end{tabular}


Table A10: Approximate polynomial solutions for Example $2(n=5)$

\begin{tabular}{c|c}
\hline Polynomials & Approximate polynomial solutions $(\tilde{\varphi}(x))$ \\
\hline \multirow{2}{*}{ BF } & $1.25424892-6.2712446 x+53.3211044 x^{2}-164.4280359 x^{3}+260.311097 x^{4}$ \\
& $-224.740958 x^{5}+116.9850364 x^{6}-49.6143677 x^{7}+19.0062071 x^{8}$ \\
& $-3.27973169 x^{9}+0.36333659 x^{10}$ \\
\hline CH & $1.09165+2.70894 x^{2}-1.95453 x^{4}+0.367927 x^{6}+0.641343 x^{8}-0.095846 x^{10}$ \\
\hline CL & $1.00552+0.79376 x+2.32544 x^{2}-6.3448 x^{3}+11.143 x^{4}-9.2554 x^{5}+3.25266 x^{6}$ \\
& $-0.050175 x^{7}-0.182068 x^{8}+0.0283702 x^{9}-0.0011348 x^{10}$ \\
\hline FL & $1.00182+0.94212 x+0.9369 x^{2}-1.14966 x^{3}+1.91422 x^{4}-1.32656 x^{5}+0.522955 x^{6}$ \\
& $-0.151293 x^{7}+0.0293789 x^{8}-0.00310813 x^{9}+0.000124325 x^{10}$ \\
\hline \multirow{2}{*}{ LLE } & $1.014+0.4905 x+4.922 x^{2}-15.74 x^{3}+28.29 x^{4}-26.0 x^{5}+11.94 x^{6}-2.361 x^{7}+0.1566 x^{8}$ \\
& $-0.001385 x^{9}+0.0000554 x^{10}$ \\
\hline
\end{tabular}

Table A11: Numerical results for Example $3(n=2)$

\begin{tabular}{c|c|c|c|c|c|c}
\hline$x$ & EXACT & BF & CH & CL & FL & LLE \\
\hline 0.0 & 1.000000 & 1.324201 & 1.115579 & 1.308356 & 0.962619 & 1.925268 \\
\hline 0.1 & 1.105171 & 1.149250 & 1.137576 & 1.140965 & 1.104778 & 1.326699 \\
\hline 0.2 & 1.221403 & 1.127303 & 1.202765 & 1.136971 & 1.235658 & 1.114158 \\
\hline 0.3 & 1.349859 & 1.220502 & 1.308749 & 1.248972 & 1.364328 & 1.168164 \\
\hline 0.4 & 1.491825 & 1.394063 & 1.451533 & 1.435142 & 1.498791 & 1.383298 \\
\hline 0.5 & 1.648721 & 1.616279 & 1.625519 & 1.659233 & 1.645981 & 1.668195 \\
\hline 0.6 & 1.822119 & 1.858518 & 1.823518 & 1.890572 & 1.811768 & 1.945545 \\
\hline 0.7 & 2.013753 & 2.095223 & 2.036734 & 2.104066 & 2.000953 & 2.152095 \\
\hline 0.8 & 2.225541 & 2.303911 & 2.254778 & 2.280195 & 2.217269 & 2.238650 \\
\hline 0.9 & 2.459603 & 2.465177 & 2.465661 & 2.405017 & 2.463385 & 2.170069 \\
\hline 1.0 & 2.718282 & 2.562688 & 2.655796 & 2.470168 & 2.740901 & 1.925268 \\
\hline
\end{tabular}

Table A12: Numerical results for Example $3(n=3)$

\begin{tabular}{c|c|c|c|c|c|c}
\hline$x$ & EXACT & BF & CH & CL & FL & LLE \\
\hline 0.0 & 1.000000 & 1.278844 & 1.084872 & 1.039803 & 0.997773 & 1.101115 \\
\hline 0.1 & 1.105171 & 1.071526 & 1.113146 & 1.094629 & 1.105565 & 1.079046 \\
\hline 0.2 & 1.221403 & 1.115273 & 1.194925 & 1.208269 & 1.222673 & 1.189416 \\
\hline 0.3 & 1.349859 & 1.287276 & 1.321589 & 1.349423 & 1.350513 & 1.350301 \\
\hline 0.4 & 1.491825 & 1.501323 & 1.480482 & 1.501606 & 1.491237 & 1.517718 \\
\hline 0.5 & 1.648721 & 1.704772 & 1.657481 & 1.659529 & 1.647277 & 1.676244 \\
\hline 0.6 & 1.822119 & 1.876199 & 1.840591 & 1.825764 & 1.820935 & 1.830375 \\
\hline 0.7 & 2.013753 & 2.023732 & 2.024562 & 2.007707 & 2.014001 & 1.996647 \\
\hline 0.8 & 2.225541 & 2.184061 & 2.216542 & 2.214821 & 2.227411 & 2.196498 \\
\hline 0.9 & 2.459603 & 2.422127 & 2.442752 & 2.456176 & 2.460938 & 2.449893 \\
\hline 1.0 & 2.718282 & 2.831494 & 2.756182 & 2.738276 & 2.712925 & 2.769683 \\
\hline
\end{tabular}


Table A13: Numerical results for Example $3(n=4)$

\begin{tabular}{c|c|c|c|c|c|c}
\hline$x$ & EXACT & BF & CH & CL & FL & LLE \\
\hline 0.0 & 1.000000 & 1.244519 & 1.067028 & 1.005244 & 1.001463 & 1.013360 \\
\hline 0.1 & 1.105171 & 1.034739 & 1.101384 & 1.103086 & 1.104569 & 1.099890 \\
\hline 0.2 & 1.221403 & 1.154437 & 1.197524 & 1.221416 & 1.221248 & 1.221631 \\
\hline 0.3 & 1.349859 & 1.359657 & 1.337214 & 1.351396 & 1.350291 & 1.353768 \\
\hline 0.4 & 1.491825 & 1.539598 & 1.497925 & 1.492578 & 1.492232 & 1.493522 \\
\hline 0.5 & 1.648721 & 1.676229 & 1.662302 & 1.647938 & 1.648659 & 1.646591 \\
\hline 0.6 & 1.822119 & 1.805699 & 1.826778 & 1.820819 & 1.821668 & 1.818975 \\
\hline 0.7 & 2.013753 & 1.979618 & 2.004689 & 2.013433 & 2.013414 & 2.013227 \\
\hline 0.8 & 2.225541 & 2.224443 & 2.217937 & 2.226605 & 2.225781 & 2.228179 \\
\hline 0.9 & 2.459603 & 2.497477 & 2.469875 & 2.460457 & 2.460113 & 2.461319 \\
\hline 1.0 & 2.718282 & 2.638158 & 2.690828 & 2.715751 & 2.717015 & 2.712973 \\
\hline
\end{tabular}

Table A14: Numerical results for Example $3(n=5)$

\begin{tabular}{c|c|c|c|c|c|c}
\hline$x$ & EXACT & BF & CH & CL & FL & LLE \\
\hline 0.0 & 1.000000 & 1.217698 & 1.055374 & 1.000455 & 0.999877 & 1.001456 \\
\hline 0.1 & 1.105171 & 1.027799 & 1.095602 & 1.105068 & 1.105212 & 1.104824 \\
\hline 0.2 & 1.221403 & 1.204613 & 1.203637 & 1.221535 & 1.221371 & 1.221828 \\
\hline 0.3 & 1.349859 & 1.388424 & 1.349121 & 1.349853 & 1.349835 & 1.349872 \\
\hline 0.4 & 1.491825 & 1.511544 & 1.502509 & 1.491715 & 1.491848 & 1.491468 \\
\hline 0.5 & 1.648721 & 1.628954 & 1.653403 & 1.648718 & 1.648752 & 1.648663 \\
\hline 0.6 & 1.822119 & 1.800016 & 1.815297 & 1.822216 & 1.822114 & 1.822420 \\
\hline 0.7 & 2.013753 & 2.025208 & 2.008863 & 2.013766 & 2.013719 & 2.013865 \\
\hline 0.8 & 2.225541 & 2.248195 & 2.232277 & 2.225451 & 2.225534 & 2.225283 \\
\hline 0.9 & 2.459603 & 2.439381 & 2.457459 & 2.459648 & 2.459643 & 2.459625 \\
\hline 1.0 & 2.718282 & 2.782557 & 2.738509 & 2.718089 & 2.718188 & 2.718006 \\
\hline
\end{tabular}

Table A15: Approximate polynomial solutions for Example $3(n=5)$

\begin{tabular}{c|r}
\hline Polynomials & Approximate polynomial solutions $(\tilde{\varphi}(x))$ \\
\hline \multirow{2}{*}{ BF } & $1.217698255-6.08849127 x+58.792735 x^{2}-199.319805 x^{3}+329.217429 x^{4}$ \\
& $-268.840521 x^{5}+113.8964514 x^{6}-47.3745451 x^{7}+23.9235674 x^{8}$ \\
& $-2.98978067 x^{9}+0.347819639 x^{10}$ \\
\hline CH & $1.0553736+4.1374469 x^{2}-11.703857 x^{4}+24.242969 x^{6}-23.550553 x^{8}+8.557129 x^{10}$ \\
\hline \multirow{2}{*}{ CL } & $1.000454+0.974295 x+0.857404 x^{2}-1.929012 x^{3}+6.3477 x^{4}-10.55566 x^{5}$ \\
& $+10.04022 x^{6}-5.23671 x^{7}+1.373203 x^{8}-0.1602158 x^{9}$ \\
& $+0.00640863 x^{10}$ \\
\hline \multirow{2}{*}{ FL } & $0.999876+1.006006 x+0.42883 x^{2}+0.52035 x^{3}-0.861173 x^{4}+1.306827 x^{5}$ \\
& $-1.093587 x^{6}+0.542844 x^{7}-0.1490253 x^{8}+0.01795593 x^{9}$ \\
LLE & $1.001456+0.918849 x+1.61144 x^{2}-6.25213 x^{3}+19.0753 x^{4}-31.4524 x^{5}+29.5633 x^{6}$ \\
& $-15.3024 x^{7}+4.00281 x^{8}-0.466882 x^{9}+0.0186753 x^{10}$ \\
\hline
\end{tabular}

